\title{
Linearized Richtmyer-Meshkov flow analysis for impulsively accelerated incompressible solids
}

\author{
A. López Ortega, D. J. Hill, D. I. Pullin, and D. I. Meiron \\ Graduate Aerospace Laboratories, California Institute of Technology, Pasadena, California 91125, USA
}

(Received 10 March 2010; published 11 June 2010)

\begin{abstract}
We present an analytical study of the linearized impulsive Richtmyer-Meshkov flow for incompressible elastic solids. Seminumerical prior investigations of a related shock-driven compressible elastic problem suggest that the interface amplitude remains bounded in time, in contrast to the unstable behavior found for gases. Our approach considers a base unperturbed flow and a linearization of the conservation equations around the base solution. The resulting initial and boundary value problem is solved using Laplace transform techniques. Analysis of the singularities of the resultant function in the Laplace domain allows us to perform a parametric study of the behavior of the interface in time. We identify two differentiated long-term patterns for the interface, which depends on the material properties: standing wave and oscillating decay. Finally, we present results for the vorticity distribution, which show that the shear stiffness of the solids is responsible both for the stabilization of the interface, and also for the period of the interface oscillations. Comparisons with previous results are discussed.
\end{abstract}

DOI: 10.1103/PhysRevE.81.066305

PACS number(s): 47.20.-k, 46.15.Ff

\section{INTRODUCTION}

The original Richtmyer-Meshkov problem [1,2] describes the instability, caused by the passage of a shock wave, of the interface separating two fluids. This problem has been the subject of study in many fields [3], including inertial confinement fusion [4], astrophysical phenomena [5], magnetohydrodynamics [6], and solid mechanics [7].

In the field of solid mechanics, Plohr and Plohr [7] performed an analysis based on linearized compressible elasticity, using free-slip boundary conditions and neo-Hookean stress-strain relations. The numerical solution of the resulting equations for a discrete range of initial conditions and material parameters revealed that the behavior of the interface separating two solids was oscillatory and stable after the shock wave-interface interaction, with shear waves advecting vorticity from the interface.

Piriz et al. [8] performed both local analysis and simulations to derive an approximate result for the long-time behavior of the interface. They provide a long-time approximation for the amplitude and frequency of the interface oscillations. However, it is unclear what boundary conditions exactly were applied.

Richtmyer-Meshkov flow has similarities with other types of flows that are unstable for fluids, such as the RayleighTaylor problem. In the field of solid mechanics, this flow has been studied, too. Plohr and Sharp in 1998 [9] used Laplace transform methods to study the instability of an incompressible metal plate to derive an expression for the critical wavelength beyond which the flow is unstable. They also demonstrated the existence of shear waves in the solids, which dissipate the vorticity deposited at the interface by an impulse. Other contributions come from Piriz et al. [10] and Terrones [11].

In this paper, we demonstrate the stability of the Richtmyer-Meshkov problem for elastic solids using an incompressible, linearized, and impulse-driven model to obtain a simple time-dependent solution. Using transform techniques, we reduce the stability calculation to an analysis of the locations of singularities in the complex plane. We show two distinct long-term types of behavior: decaying oscillations and standing waves.

\section{PROBLEM DESCRIPTION}

The impulsive Richtmyer-Meshkov problem for solids is formulated using small perturbation theory and twodimensional plane-strain conditions for both materials. Figure 1 shows the initial configuration and the parameters that influence the problem. The shock wave that initiates the motion of the system in the compressible Richtmyer-Meshkov problem is replaced with an impulsive acceleration $V \delta(t)$. The interface is described as perpendicular to the direction of the impulse with the addition of a sinusoidal perturbation of wavelength $2 \pi / k$ and initial amplitude $\eta_{0}$. Both materials $(j=-,+)$ are described by their density $\rho_{j}$ and their shear wave speeds $c_{j}=\sqrt{\mu_{j} / \rho_{j}}$, where $\mu_{j}$ is the material shear modulus. The materials are taken to be purely elastic and incompressible.

Mass conservation and momentum equations are applied to each material in order to obtain a system of partial differ-

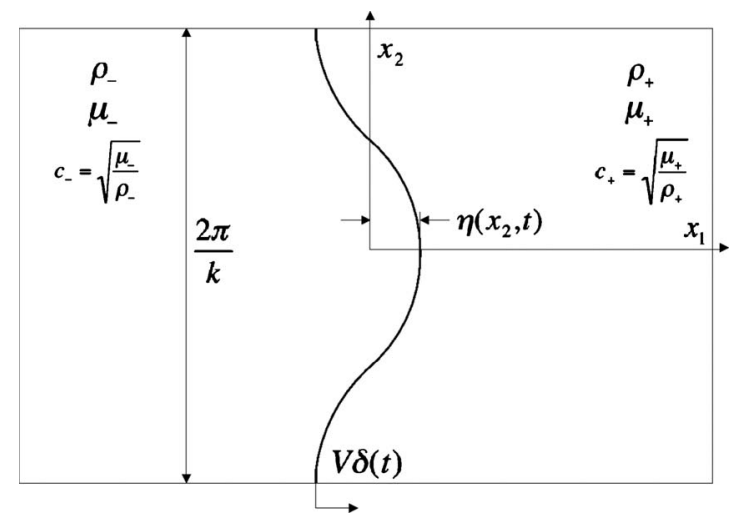

FIG. 1. Description of the problem. The interface is $x_{1}$ $=\eta\left(x_{2}, t\right)$. Left and right states are denoted by "-" and "+," respectively. 
ential equations for evolution of the perturbed flow, while boundary conditions relate the velocities and stresses at the interface between materials. The system is transformed in time by a Laplace transform, and in one spatial dimension by a normal-mode assumption. The resulting fourth-order ordinary differential equation for the transform of the velocity field in each material is then solved. From this expression, other useful variables, such as amplitude of the interface and the vorticity, can be calculated.

\section{EQUATIONS OF MOTION AND BOUNDARY CONDITIONS}

We describe here the general form of the conservation equations along with the incompressibility and plane-strain constraints. These equations are particularized in the following sections to describe the base and perturbed flows.

Mass conservation and conservation of linear momentum are expressed in Eulerian form as

$$
\begin{gathered}
\frac{\partial u_{i}}{\partial x_{i}}=0, \\
\rho \frac{\partial u_{i}}{\partial t}+\rho u_{j} \frac{\partial u_{i}}{\partial x_{j}}-\frac{\partial \sigma_{i j}}{\partial x_{j}}=0,
\end{gathered}
$$

where $u_{i}$ is the velocity in the $x_{i}$ direction and $\sigma_{i j}$ is the Cauchy stress tensor. The kinematic equation that describes the evolution of the interface $\eta\left(x_{2}, t\right)-x_{1}=0$ is given by

$$
\frac{D}{D t}\left[\eta\left(x_{2}, t\right)-x_{1}\right]=0,
$$

where $D / D t=\partial / \partial t+u_{j} \partial / \partial x_{j}$ is the material derivative.

Additional kinematics of solid deformation are described by a gradient of a Lagrangian map $\mathbf{x}(\mathbf{X}, t)$, where $\mathbf{x}$ is the location of the particle at time $t$ that was originally at point $\mathbf{X}$. An inverse map is defined as $\mathbf{X}=\mathbf{X}(\mathbf{x}, t)$. From these relationships two tensors are obtained: the deformation tensor $F_{i j}=\partial x_{i} / \partial X_{j}$ and the inverse deformation tensor $g_{i j}=\partial X_{i} / \partial x_{j}$. These two tensors are related by $F_{i j} g_{j k}=\delta_{i k}$. Components $g_{i j}$ evolve according to

$$
\frac{\partial g_{i j}}{\partial t}+\frac{\partial}{\partial x_{j}}\left(g_{i k} u_{k}\right)=0 .
$$

The assumptions of plane strain and incompressiblitiy impose relations between the elements of the inverse deformation tensor $g_{i j}$. From the two dimensionality of plane strain we have

$$
g_{13}=g_{23}=g_{31}=g_{32}=0, \quad g_{33}=1 .
$$

This may be combined with conservation of mass expressed as $\operatorname{det}(\mathbf{g})=\rho / \rho_{o}$ to give

$$
g_{11} g_{22}-g_{21} g_{12}=1,
$$

for an incompressible material.

To close the problem, we define a constitutive law relating stresses to the material deformation. We model both materials with a neo-Hookean constitutive relation,

$$
\sigma_{i j}=-p \delta_{i j}+\mu F_{i k} F_{j k}
$$

where pressure $p$ is a Lagrange multiplier that enforces incompressibility, i.e., $p$ satisfies

$$
\frac{\partial^{2} p}{\partial x_{i}^{2}}=\frac{\partial^{2}}{\partial x_{i} \partial x_{j}}\left(-\rho u_{i} u_{j}+\mu F_{i k} F_{j k}\right),
$$

for uniform $\rho$.

\section{Boundary conditions}

Boundary conditions at the interface $\eta$ relate variables from each material. Defining $\mathbf{n}$ and $\mathbf{t}$ as the unit normal and tangent at the boundary, the four boundary conditions are, respectively, continuity of normal velocity, continuity of normal stress, and zero tangential stress (i.e., free slip),

$$
\begin{gathered}
{\left[\left|u_{i}\right|\right] n_{i}=0,} \\
n_{i}\left[\left|\sigma_{i j}\right|\right] n_{j}=0, \\
t_{i} \sigma_{i j}^{-} n_{j}=0=t_{i} \sigma_{i j}^{+} n_{j},
\end{gathered}
$$

where $[|q|]$ indicates the jump $q^{+}-q^{-}$. Boundary conditions at infinity are imposed as radiation conditions, requiring all waves at infinity to be outgoing.

\section{BASE FLOW AND LINEARIZATION}

The trivial base flow assumes a planar interface aligned with the $x_{2}$ axis and perpendicular to the direction of the impulse. The resulting one-dimensional motion depends on $x_{1}, t$ only and does not deform the material. The reference frame is chosen with the unperturbed interface located at $x_{1}=0$ after the impulse at $t=0$, so that

$$
\eta=-V t[H(t)-1],
$$

where $V$ is the impulsive velocity and $H(t)$ is the Heaviside function. In each material the velocity, deformation, and pressure fields are given by

$$
\begin{gathered}
u_{1}=-V[H(t)-1], \\
u_{2}=0, \\
g_{i j}=\delta_{i j}, \\
p=\rho V \delta(t) x+\mu,
\end{gathered}
$$

where the resulting Cauchy stress $\sigma_{i j}=-\rho V \delta(t) x \delta_{i j}$ represents the fact that for this special geometry the material is in an unstressed state before and after the impulse.

\section{Linearization}

Once the base flow variables are known, the complete value of each variable $q$ defining the problem is calculated adding a perturbation term $q^{\prime}$ to the base flow result $\bar{q}$, i.e., Eqs. (12)-(16). Substituting $q=\bar{q}+q^{\prime}$ in the equations of motion [Eqs. (1)-(4)] and retaining terms that are at most linear 
in perturbation quantities gives the following system of linear equations for the perturbed variables:

$$
\begin{gathered}
\frac{\partial u_{j}^{\prime}}{\partial x_{j}}=0, \\
\rho \frac{\partial u_{j}^{\prime}}{\partial t}+\overline{u_{1}} \frac{\partial u_{i}^{\prime}}{\partial x_{1}}-\frac{\partial \sigma_{i j}^{\prime}}{\partial x_{i}}=0, \\
\frac{\partial \eta^{\prime}}{\partial t}-u_{1}^{\prime}=0, \\
\frac{\partial g_{i j}^{\prime}}{\partial t}+\overline{u_{1}} \frac{\partial g_{i 1}^{\prime}}{\partial x_{j}}+\frac{\partial u_{i}^{\prime}}{\partial x_{j}}=0,
\end{gathered}
$$

where $\overline{u_{1}}$ is given by Eq. (13).

Linearization of the neo-Hookean stress-strain relations [Eq. (7)] produces

$$
\sigma_{i j}^{\prime}=-p^{\prime} \delta_{i j}-\mu\left(g_{i j}^{\prime}+g_{j i}^{\prime}\right),
$$

the familiar relation for a Hookean material of linear elasticity with Lamé's first parameter $\lambda=0$. The boundary conditions for the normal velocity, normal stress, and free-slip conditions [Eqs. (9)-(11)], respectively, become

$$
\begin{gathered}
{\left[\left|u_{1}^{\prime}\right|\right]=0,} \\
{\left[\left|\sigma_{11}^{\prime}\right|\right]=-[|\rho|] V \eta^{\prime} \delta(t),} \\
\sigma_{21}^{\prime-}=0=\sigma_{21}^{\prime+},
\end{gathered}
$$

and are applied on the unperturbed interface (i.e., at $x_{1}=0$ for $t \geq 0$ ).

\section{SOLUTION OF THE PROBLEM}

We assume now that the interfacial perturbation is initially sinusoidal in $x_{2}$ and the resulting perturbation solutions may be expressed as Fourier modes in $x_{2}, q^{\prime}\left(t, x_{1}, x_{2}\right)$ $=\hat{q}\left(x_{1}, t\right) \exp \left(i k x_{2}\right)$. In addition, the differential expressions containing time dependence are converted to algebraic relations using the Laplace transform. The initial conditions for the perturbations precede the impulse and are thus zero.

Denoting the Laplace transforms of a variable $\hat{q}$ with capital letters (e.g., $G_{i j}=\mathcal{L}\left[\hat{g}_{i j}\right]$ ), the linearized equations (17)-(20) become

$$
\begin{gathered}
\frac{d}{d x} U_{1}+i k U_{2}=0, \\
\rho s U_{1}+2 \mu \frac{d}{d x} G_{11}+\frac{d}{d x} P+\mu i k\left(G_{12}+G_{21}\right)=0, \\
\rho s U_{2}+2 \mu i k G_{22}+i k P+\mu \frac{d}{d x}\left(G_{12}+G_{21}\right)=0, \\
s G_{i 1}+\frac{d}{d x} U_{i}=0,
\end{gathered}
$$

$$
s G_{i 2}+i k U_{i}=0 .
$$

This system of equations is manipulated to obtain an expression for $U_{1}$, which is a fourth-order ordinary differential equation to be solved separately in each material (i.e., $x<0$ and $x>0$ ),

$$
\frac{d^{4}}{d x^{4}} U_{1}-\left(\frac{s^{2}}{c^{2}}-2 k^{2}\right) \frac{d^{2}}{d x^{2}} U_{1}+\left(\frac{k^{2} s^{2}}{c^{2}}+k^{4}\right) U_{1}=0,
$$

where $c^{2}=\mu / \rho$. The general solution for $U_{1}$ is spanned by the fundamental solutions

$$
\left\{e^{k x_{1}}, e^{-k x_{1}}, e^{\sqrt{\left(s^{2} / c^{2}\right)+k^{2}} x_{1}}, e^{-\sqrt{\left(s^{2} / c^{2}\right)+k^{2}} x_{1}}\right\} .
$$

Apart from the four boundary conditions imposed at the interface (22)-(24), regular boundary conditions at infinity are imposed after a suitable definition of the generally multivalued square root. Recalling that the inverse Laplace transform is defined by a vertical contour of integration in the complex $s$ plane located to the right of all singularities, we explicitly define the square roots to have positive real parts in the right half plane. Boundary conditions at $x \rightarrow \pm \infty$ then imply

$$
U_{1}^{ \pm}\left(s, x_{1}\right)=A_{ \pm}(s) e^{\mp k x_{1}}+B_{ \pm}(s) e^{\mp \sqrt{\left(s^{2} / c_{ \pm}^{2}\right)+k^{2}} x_{1}},
$$

where the upper sign is taken for $x>0$ and the lower sign for $x<0$.

The four unknowns $A_{ \pm}(s), B_{ \pm}(s)$ are determined by application of the four interface boundary condition equations transformed into Laplace space. Solving the resulting linear system gives

$$
\begin{gathered}
A_{ \pm}(s)=\frac{(r-1) V \eta_{0} k s\left(2 c_{ \pm}^{2} k^{2}+s^{2}\right)}{\Omega(s)}, \\
B_{ \pm}(s)=-\frac{(r-1) V \eta_{0} k s 2 c_{ \pm}^{2} k^{2}}{\Omega(s)},
\end{gathered}
$$

where $\Omega(s)$ is given by

$$
\begin{aligned}
\Omega(s)= & (1+r) s^{4}+4 c_{-}^{2} k^{2} s^{2}+4 c_{+}^{2} k^{2} r s^{2}-4 c_{-}^{4} k^{4} \\
& \times\left(-1+\sqrt{1+\frac{s^{2}}{c_{-}^{2} k^{2}}}\right)-4 c_{+}^{4} k^{4} r\left(-1+\sqrt{1+\frac{s^{2}}{c_{+}^{2} k^{2}}}\right),
\end{aligned}
$$

and $r=\rho_{+} / \rho_{-}$. With this, the problem is solved in the complex $s$ plane: all other transformed variables may be obtained from $U_{1}\left(x_{1}, s\right)$ by manipulation of Eqs. (25)-(29).

Before analyzing the solutions in detail, we obtain physical insight by examining the evolution of vorticity, defined for a two-dimensional flow as $\omega=\partial u_{2} / \partial x_{1}-\partial u_{1} / \partial x_{2}$. By taking the curl of the linearized momentum (18) to eliminate the hydrodynamic pressure and then differentiating in time to expresses the time derivative of the stress in terms of the rate of strain with the aid of Eq. (20), one finds that the vorticity due to the linear perturbations satisfies the second-order wave equation, 


$$
\frac{\partial^{2} \omega^{\prime}}{\partial t^{2}}=c^{2} \frac{\partial^{2} \omega^{\prime}}{\partial x_{i}^{2}}
$$

in each material for $t>0$. The fourth-order ordinary differential equation (ODE) [Eq. (30)] is simply the Laplace transform of this equation cast in terms of $\hat{u}_{1}$ after the normalmode assumption has been made: $u^{\prime}\left(t, x_{1}, x_{2}\right)$ $=\hat{u}\left(t, x_{1}\right) \exp \left(i k x_{2}\right)$.

Using incompressibility (17), vorticity can be expressed solely in terms of the velocity perturbation $\widehat{u_{1}}$ as

$$
\omega^{\prime}\left(t, x_{1}, x_{2}\right)=\hat{\omega}\left(t, x_{1}\right) e^{i k x_{2}}=i\left(-k \hat{u}_{1}+\frac{1}{k} \frac{\partial^{2} \hat{u}_{1}}{\partial x_{1}^{2}}\right) e^{i k x_{2}}
$$

From this one may readily verify that the $A_{ \pm}(s)$ solution in Eq. (32) corresponds to an irrotational velocity field, while the $B_{ \pm}(s)$ solution identifies shear waves. Note that expression (37) holds within each solid but not at the interface, where a finite jump of the tangential velocity $u_{2}$ produces an additional vortex sheet. The Laplace transform of Eq. (37) may be written as

$$
\omega_{ \pm}\left(s, x_{1}\right)=-2 i k U_{1}(s, 0) e^{\mp \sqrt{\left(s^{2} / c_{ \pm}^{2} k^{2}\right)+1} k x_{1}},
$$

where $U_{1}(s, 0)=U_{1}^{+}(s, 0)=U_{1}^{-}(s, 0)$ is the transform of the normal velocity at the interface. Excluding the vortex sheet the remaining vorticity $\omega$ is continuous at the interface.

Using the convolution theorem, the vorticity mode in physical space $\hat{\omega}\left(t, x_{1}\right)$ can be computed as

$$
\hat{\omega}_{ \pm}\left(x_{1}, t\right)=\int_{0}^{t} \hat{\omega}\left(\tau, x_{1}=0\right) \hat{g}_{ \pm}\left(t-\tau, x_{1}\right) d \tau,
$$

where $\quad \hat{g}_{ \pm}\left(t, x_{1}\right)=\mathcal{L}^{-1}\left[\exp \left\{\mp \sqrt{\left(s^{2} / c_{ \pm}^{2} k^{2}\right)+1} k x_{1}\right\}\right] \quad$ and $\hat{g}_{ \pm}\left(t, x_{1}\right) \exp \left(i k x_{2}\right)$ satisfies the second-order wave equation (36) as outward radiating waves. This convolution expression shows how the vorticity that is deposited at the interface is transported by the shear waves into the materials for $t>0$.

\section{ANALYSIS OF THE INTERFACE BEHAVIOR}

To determine the time-dependent behavior of the velocity field, interface amplitude, or other quantities of interest, the inverse Laplace transform of the $s$-dependent expressions is performed. The Bromwich integral offers a general way of performing this operation,

$$
f(t)=\mathcal{L}^{-1}\{F(s)\} \equiv \frac{1}{2 \pi i} \lim _{T \rightarrow \infty} \int_{\gamma-i T}^{\gamma+i T} e^{s t} F(s) d s,
$$

where $\gamma$ is a real value situated to the right of all singularities of the function $F(s)$.

Expression (40) can be calculated numerically to obtain the complete evolution of the interface $\eta$ in time and such results are reported in the next section. Here, we use the tools of complex analysis and asymptotics to examine analytically both the initial growth rate and the long-term behavior of the interface.

\section{A. Short-time interface behavior}

From Eq. (19) [i.e., $\left.\partial \eta^{\prime} / \partial t=u_{1}^{\prime}\left(0, x_{2}, t\right)\right]$, we see that the growth rate of the linearized interface may be computed by calculating the inverse Laplace transform of $U_{1}\left(s, x_{1}=0\right)$. Using Eq. (32), this quantity may be computed as

$$
\frac{\partial \hat{\eta}}{\partial t}=\mathcal{L}^{-1}\left[\frac{(r-1) V \eta_{0} k s^{3}}{\Omega(s)}\right] .
$$

We can recover the familiar impulsive Richtmyer-Meshkov result for inviscid fluids by eliminating the shear strength $\mu_{ \pm}$ of the materials (i.e., $\left.c_{ \pm}=0\right)$. This reduces $\Omega$ to $\Omega=(r+1) s^{4}$ and the inverse Laplace transform gives

$$
\frac{\partial \hat{\eta}}{\partial t}=\frac{(r-1)}{(r+1)} V \eta_{0} k
$$

from which one sees that the perturbation amplitude grows linearly in time. The ratio $(r-1) /(r+1)$ is the Atwood number $A_{t}$.

For the case of elastic solids, the initial growth rate for any value of shear strength is also given by Eq. (42) as can be seen by examining the large $s$ limit $U_{1}(0, s) \sim A_{t} V \eta_{0} k / s$. Physically for times much shorter than the characteristic shear wave speeds $t \ll 1 /(k c)$ the vorticity is located approximately at the interface and the interface initially evolves as in the fluid example.

\section{B. Long-time interface behavior}

We now perform an analysis of the time-dependent behavior of the interface amplitude $\hat{\eta}(t)$ directly. From Eq. (41) we see that

$$
\hat{\eta}(t)=\eta_{o}+\mathcal{L}^{-1}[N(s)]
$$

where

$$
N(s)=\frac{(r-1) V \eta_{0} k s^{4}}{\Omega(s)},
$$

and $\Omega(s)$ is as defined in Eq. (35). In performing the inverse transform, the analytical properties of the denominator $\Omega$ determine the time dependence of the solution. With the aim of obtaining the long-time behavior of the interface, we construct a specific representation of $\Omega$ and deform the Bromwich integral around the possible singularities.

While the square roots in $\Omega$ have been defined to have a positive real part in $\operatorname{Re}(s)>0$, there are several possibilities for the specific locations of the branch cuts. Figure 2 shows the set of branch cuts that were used for our analysis. This choice offers the possibility of applying linearization techniques to calculate the long-term behavior, as is shown later in this section.

Using this disposition of branch cuts, the Bromwich integral for $\eta$ can be closed in the left half plane for $t>0$ and computed as 


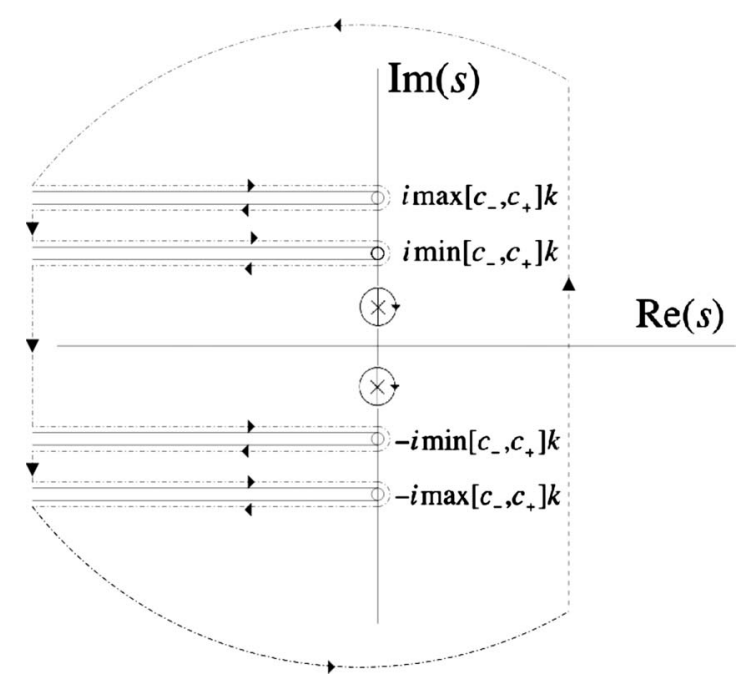

FIG. 2. Set of branch cuts used for the analysis and deformation of the Bromwich integral. We show two poles in the imaginary axis, which is not a general case. For any pole found, the contour is deformed to surround it.

$$
\begin{aligned}
\hat{\eta}(t)= & \eta_{o}+\frac{1}{2 \pi i} \sum_{n} \operatorname{Res}\left(N\left(s_{n}\right)\right) e^{s_{n} t} \\
& +\frac{1}{2 \pi i} \sum_{l=-}^{+} \int_{0}^{\infty} e^{-x t+i c_{l} t}\left[N\left(-x+i c_{l}^{b} k\right)-N\left(-x+i c_{l}^{a} k\right)\right] d x \\
& +\frac{1}{2 \pi i} \sum_{l=-}^{+} \int_{0}^{\infty} e^{-x t-i c_{l} t}\left[N\left(-x-i c_{l}^{b} k\right)-N\left(-x-i c_{l}^{a} k\right)\right] d x,
\end{aligned}
$$

where $s_{n}$ are the possible zeros of the denominator; the superscripts " $a$ " and " $b$ " determine the value of the function right above and below of the branch cut, respectively. The part of the contour that lies at infinity has no contribution according to Jordan's lemma and the turning integrals around the branch points can be shown to contribute nothing as their radius goes to zero. Hence, the behavior of the interface is determined by the residue of the amplitude function in the complex $s$ plane at the zeros of $\Omega$ and by the integration around the branch cuts.

\section{Contributions of the branch cuts}

It can be seen directly that the oscillatory exponentials may be taken out of the integrals in Eq. (45), and in addition it can be shown that the magnitude of the integrals decays with time. This follows from the fact that functions $|N(s)|$ are, in general, bounded along the branch cuts, although there are certain discrete parametric exceptions that correspond to poles of $N(s)$ crossing a branch cut and onto a separate sheet. In such a case, the poles are simple poles and the principal values of the integrals are well defined.

The long-time asymptotic contributions from the branch integrals to $\hat{\eta}(t)$ may be computed using Watson's lemma [12]. Essentially for large $t$, an expansion of $\left[N\left(-x \pm i c^{b} k\right)\right.$ $\left.-N\left(-x \pm i c^{a} k\right)\right]$ about $x=0$ is used. Note that care must be taken in treating the various square roots with respect to the

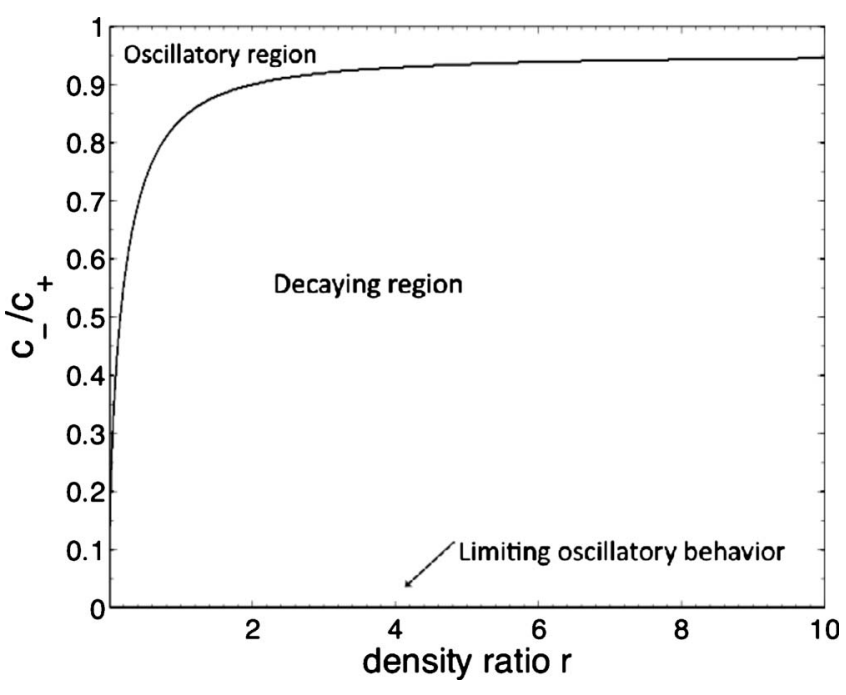

FIG. 3. Regions of existence and nonexistence of pure imaginary poles. A parametric set that lies in the first (second) region produces an oscillatory (decaying) behavior of the interface, respectively.

disposition of the branch cuts. Local to a given branch point, such a procedure gives an expression of the form

$$
N\left(-x \pm i c^{b} k\right)-N\left(-x \pm i c^{a} k\right)=\sqrt{x} \sum_{j=0}^{\infty} a_{j} x^{j},
$$

where $\sqrt{x}$ denotes the usual real square root. Then, expression (46) multiplied by $\exp (-x t)$ and integrated gives terms in $1 / t^{3 / 2}, 1 / t^{5 / 2}, 1 / t^{7 / 2}, \ldots$. When the exponentials $\exp ( \pm i c k t)$ are included, the resulting terms clearly oscillate with the shear wave frequency while decaying to zero as $t \rightarrow \infty$. The Appendix develops an approximation for the growth rate using the first term $\left(a_{0} \sqrt{x}\right)$ of this approximation.

\section{Contributions of the poles}

The denominator $\Omega$ is a single valued sheet of a multivalued function containing square roots. A rationalization is performed by forming the product of all sheets to obtain a polynomial of degree 16 . The 16 roots of this polynomial are computed numerically but only a few are real zeros of $\Omega$. A simple evaluation of our denominator at the roots allows one to determine which residues need to be computed. The number of these poles varies depending on the parameters of the problem.

Our analysis reveals that all the zeros of $\Omega$ correspond to simple poles of $N(s)$ and lie in $\operatorname{Re}(s) \leq 0$, which combined with our analysis of the branch-cut integrals indicates that the interface may be classified as stable. The positions of the poles depend on the ratio of shear wave velocities $c_{-} / c_{+}$and the density ratio $r$.

Exploring this parameter space, we find regions where there are a pair of conjugate poles that lie in the imaginary axis (oscillatory) and regions where all poles lie in $\operatorname{Re}(s)$ $<0$. Figure 3 shows the region in parameter space where the dominant poles produce pure oscillatory behavior and in the region where the poles contribute at most exponentially decaying oscillatory behavior. 


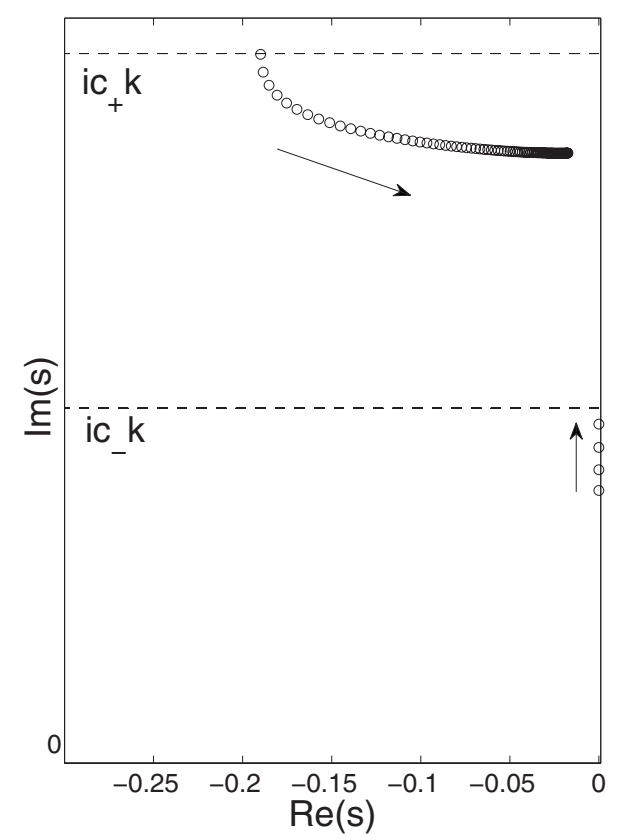

FIG. 4. Position of the closest pole with respect to the imaginary axis and relative to the branch cuts (horizontal dashed lines). Another conjugate pole lies in $\operatorname{Im}(s)<0$. Arrows show the direction the poles follow when incrementing $c_{-}$, leaving $c_{+}$and $r$ unchanged. When both velocities are similar, the poles lie on the imaginary axis and the interface oscillates. After reaching the branch cut, the pole reappears and tends to the imaginary axis as $c_{-} \rightarrow \infty$.

Oscillatory behavior is obtained when both materials have a similar shear wave speed. The region around $c_{-} / c_{+}=1$ where this happens depends on the density ratio $r$. As the ratio $c_{-} / c_{+}$separates from 1 , the pure imaginary poles travel along the imaginary axis toward the lowest pair of branch points. As this happens, the amplitude of the oscillations decreases, representing a smooth transition toward decaying oscillatory behavior. After a certain critical value of the shear wave velocity ratio, which depends on the density ratio, the poles reach the lowest branch cut and disappear; and for such parameters, the interface amplitude oscillates while decaying with time to $\eta_{o}$.

A particular case, presented in Fig. 3, that gives oscillatory behavior of the interface is that of one shear wave velocity being zero and the other finite (or one being finite and the other infinite). This case is the asymptotic result of the decaying behavior and will be explained by monitoring the location of the poles closest to the imaginary axis.

Figure 4 shows the position of the closest pole with respect to the imaginary axis and relative to the position of the branch cuts with $c_{-} \geq c_{+}$. First, the poles lie in the imaginary axis for $c_{-}=c_{+}$and they approach the lower branch cut as $c_{-}$ increases. After that, if we continue to increase $c_{-}$, the poles reappear from the superior branch cut and separate from it approaching the imaginary axis.

In conclusion, when our shear wave velocities are far from $c_{-}=c_{+}$, the behavior of the interface is decaying. As the values of the shear wave velocities separate from each other, a pair of conjugate poles tend to approach the imaginary axis. This means that the interface behavior in time looks like an oscillatory exponential decay that decays more slowly as the values of the shear wave velocity are more distinct. Finally, when one of them becomes zero, the pole reaches the imaginary axis and the behavior of the interface is oscillatory in time.

\section{Conclusions of the analysis}

We distinguish two different long-term types of behavior for the interface amplitude $\hat{\eta}(t)$ : pure oscillatory and oscillatory decay. When discussed in terms of the interface $\eta\left(t, x_{2}\right)=\hat{\eta}(t) \exp \left(i k x_{2}\right)$ these correspond to standing oscillatory waves and decaying waves.

As the branch-cut integration always results in algebraically decaying waves oscillating at the two shear speeds, the classification of the late-time behavior is a function of the locations of poles, i.e., the zeros of $\Omega$. For the oscillatory case, after an initial transient due to the branch cuts, the interfacial waves oscillate at a frequency determined by the position of the poles on the imaginary axis. The relative amplitude of this wave depends on the position of the poles too through the calculation of residues. This amplitude decreases as we parametrically approach the critical value of $c_{-} / c_{+}$. After that, the behavior changes to time-dependent decay. Physically the free-slip condition allows interfacial shear waves to persist when the shear speeds are similar in both materials, but when these nonzero speeds differ too greatly (Fig. 3) such waves decay.

The other case that gives a late-time oscillatory behavior for $\hat{\eta}(t)$ is the extreme case of one of the shear wave velocities being zero (which indicates that this material is unaffected by shear stresses, equivalent to a inviscid fluidlike material). For this case, vorticity can only be carried off the interface by the material with a nonzero shear modulus.

With respect to the oscillatory decaying behavior, we have shown that it occurs when the shear wave velocities are sufficiently different and no poles exist on the imaginary axis. To analyze this region, the position of the pair of conjugate poles that are closest to the imaginary axis is important. If the pole is very close to it (which occurs when the two shear wave velocities are very different) the subdominant exponentially decaying traveling wave may be present for some time as the characteristic decay rate of the resulting wave is very small. On the other hand, when this pair of poles is far from the imaginary axis, which happens just after reaching the critical shear wave speed ratio that separates oscillatory from decaying behavior; the evolution of the interface is controlled by the integral around the branch cuts and exhibits a $1 / t^{3 / 2}$ decay.

\section{RESULTS}

In this section, some specific results from our solution of the linearized Richtmyer-Meshkov flow are presented. We aim to visualize the general conclusions extracted from the analytical approach to the problem.

The most representative variable to discuss the stability of the system is the amplitude of the interface. For that reason, the first group of results shows the behavior of this variable 


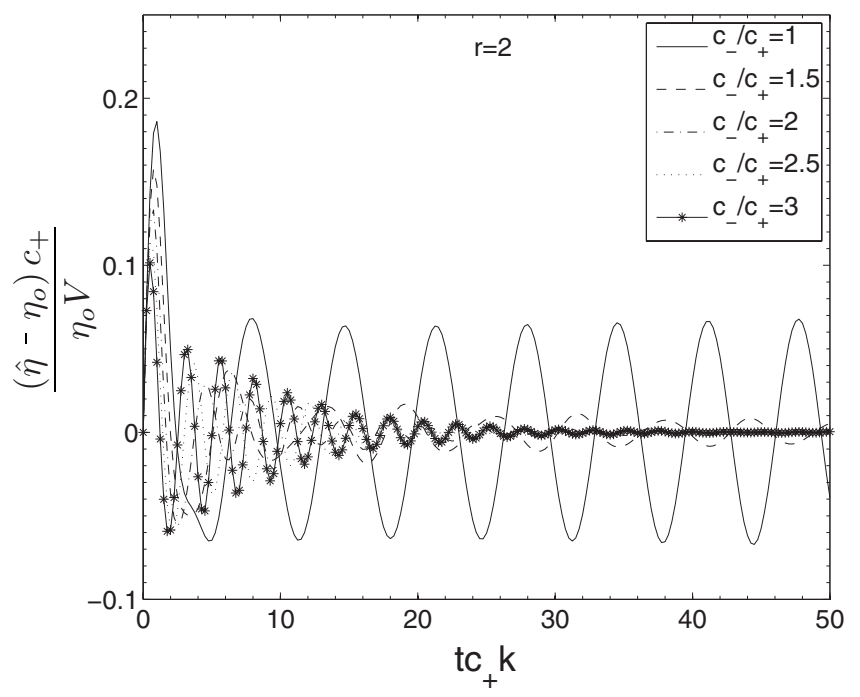

FIG. 5. Interface amplitude varying $c_{-} / c_{+}$from 1 to 3 and $r$.

under changes in the parameters that control the problem. A second group describes the frequency behavior of the longtime oscillations, comparing it with previously published approximate results [8]. Finally, we show the way the vorticity is carried away from the interface and the evolution of tangential slip velocity at the interface.

\section{A. Interface behavior}

Figure 5 shows the amplitude of the interface in time (conveniently nondimensionalized) when the shear wave velocity ratio $c_{-} / c_{+}$is in the range $1 \leq c_{-} / c_{+} \leq 3$, leaving the density ratio fixed. As expected, after the transient, the interface amplitude $\hat{\eta}(t)$ oscillates for the case $c_{-} / c_{+}=1$. For the other cases the interface amplitude decays faster as $c_{-} / c_{+}$is increased.

Figure 6 shows the amplitude of the interface when $c_{-} / c_{+}$ is increased from 0.2 to 1 . A practically oscillatory behavior

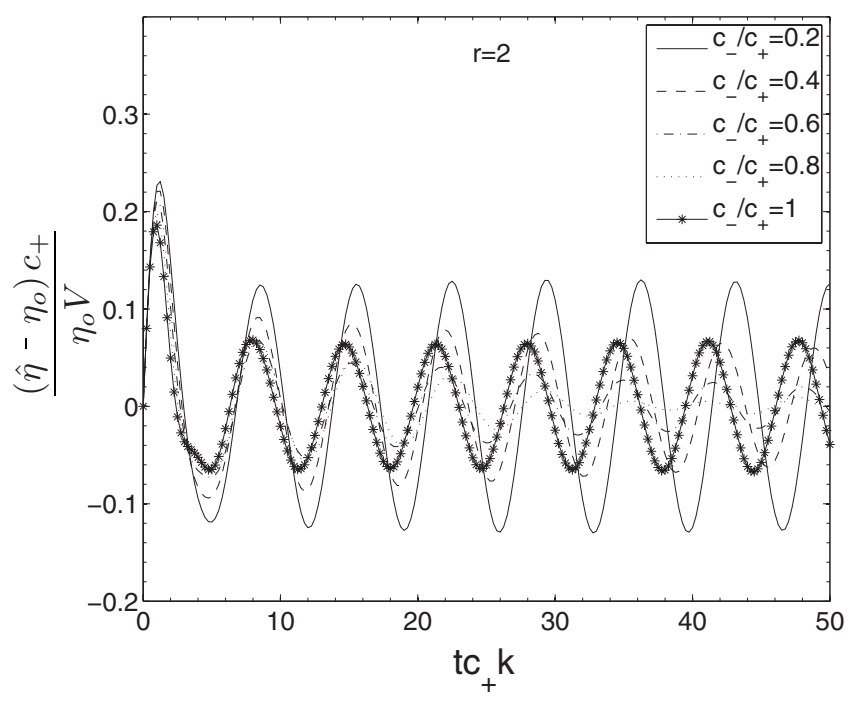

FIG. 6. Interface amplitude varying $c_{-} / c_{+}$from 1 to 3 and fixed is observed for $c_{-} / c_{+}=0.2$ and $c_{-} / c_{+}=0.4$. As we already pointed out, the interface oscillates for $c_{-} / c_{+}=0$. Therefore, values of 0.2 and 0.4 are close enough to zero to exhibit a very low rate of decay. For the intermediate values, the amplitude of the interface decays faster, but it returns to oscillatory for $c_{-} / c_{+}=1$.

\section{B. Oscillation period}

An attempt to perform a long-time $(s \rightarrow 0)$ approximation of Eq. (32) leads to the following expression:

$$
U(s, 0)=\frac{(r-1) V \eta_{0} k s}{2(1+r)\left(s+i k \sqrt{\frac{\mu_{-}+\mu_{+}}{\rho_{-}+\rho_{+}}}\right)\left(s-i k \sqrt{\frac{\mu_{-}+\mu_{+}}{\rho_{-}+\rho_{+}}}\right)} .
$$

From this last expression, we obtain that the oscillation period is

$$
\frac{T}{T_{0}}=\sqrt{\frac{1+r}{1+\frac{\mu_{+}}{\mu_{-}}}}
$$

with $T_{0}=(2 \pi / k) \sqrt{\rho_{-} / \mu_{-}}$. This value is on the order of $\max \left[c_{-}, c_{+}\right] k$, which formally contradicts our principal assumption in performing the long-time approximation, $s$ $\ll\left(c \_k, c_{+} k\right)$. As a consequence, performing an inverse Laplace transform of Eq. (47) leads to an inaccurate value for the amplitude and does not reflect a possible decaying behavior; in fact it predicts that the long-time amplitude scales as $1 / T$ for any value of $c_{-} / c_{+}$.

Piriz et al. [8] provided a similar expression

$$
\frac{T}{T_{0}}=\frac{1.55}{\sqrt{2}} \sqrt{\frac{1+r}{1+\frac{\mu_{+}}{\mu_{-}}}}
$$

obtained by a mixture of local analysis and simulation. They also predicted pure oscillation with a $1 / T$ amplitude as the long-time behavior irrespective of $c_{-} / c_{+}$. This is because the simple analytical model proposed in [8] computes the interface behavior using Newton's second law with a forcing term that is proportional to the amplitude of the interface and a mass term that is proportional to its acceleration. The resulting equivalent mass-spring system does not capture decaying oscillations.

Figures 7 and 8 show a comparison between these approximations and the result calculated from the complete model. The oscillation period arising from the pole that is closer to the imaginary axis (the imaginary part of the pole position) is also compared. In spite of the inaccuracy for calculating the expected behavior of the system (oscillating decay or pure oscillatory), the long-term approximation in fact gives a very good result for the oscillation frequency. As expected, as the ratio $c_{+} / c_{-}$separates from 1 , the oscillation period of the complete system is closer to that associated with the pole that is closer to the imaginary axis. 


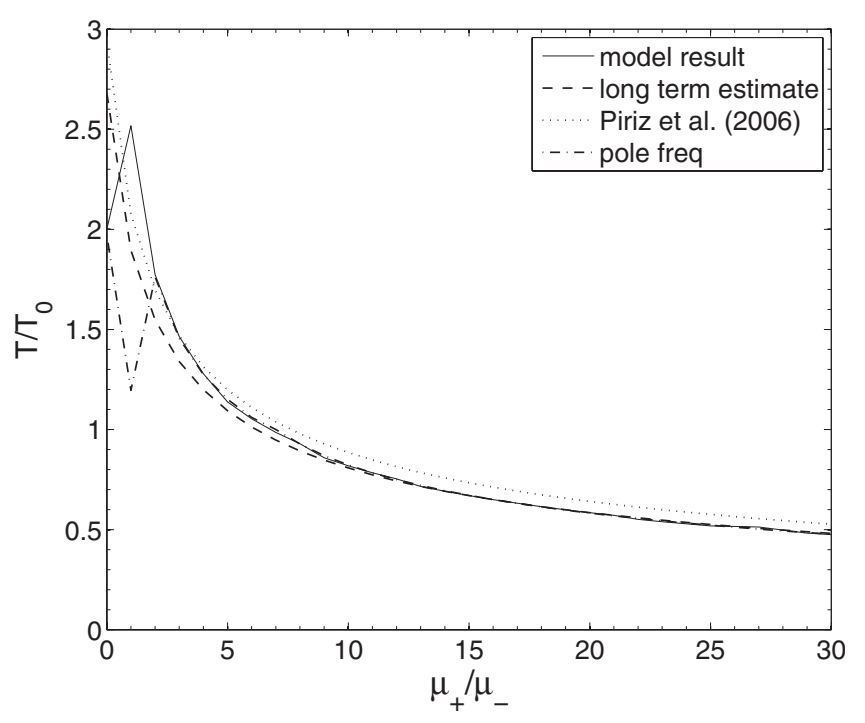

FIG. 7. Oscillation period for fixed density ratio of 6.182 and varying shear modulus. The complete model result is calculated performing numerically the inverse Laplace transform of the interface amplitude and counting the time between oscillations. The long-term estimate uses Eq. (48). The estimate of Piriz et al. uses Eq. (49). The pole frequency is calculated by locating the pole in the complex plane.

\section{Vorticity}

We have shown that vorticity is controlled by a wave equation. At $t=0$, the impulse produces a tangential velocity that is equal in magnitude and opposite in direction in the materials. For a incompressible inviscid fluid, vorticity lies at the interface and cannot be dissipated or transported. There-

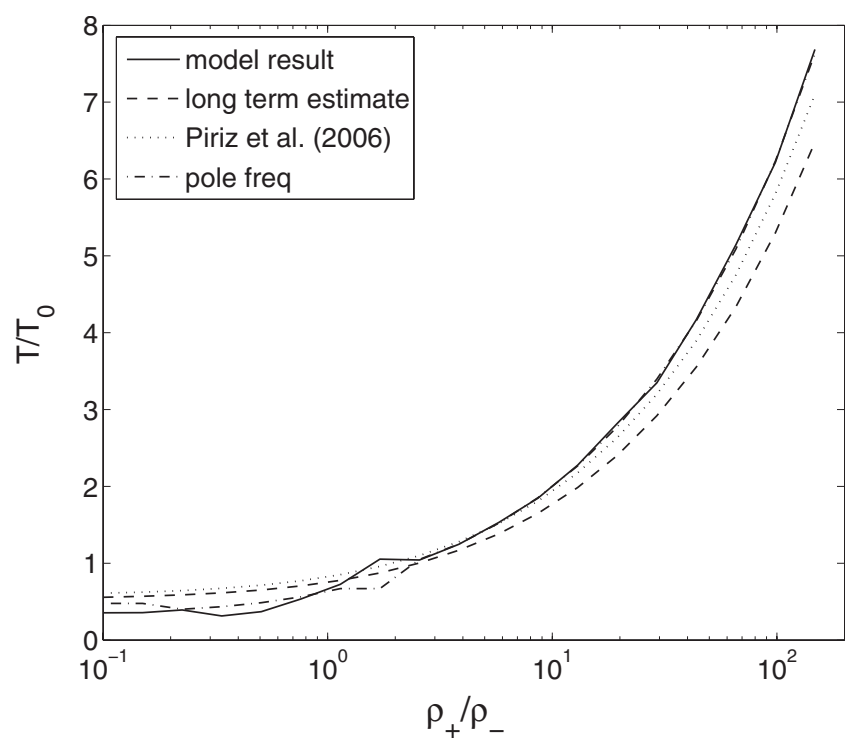

FIG. 8. Oscillation period for varying density ratio and fixed ratio of shear modulus $\mu_{+} / \mu_{-}$. The complete model result is calculated performing numerically the inverse Laplace transform of the interface amplitude and counting the time between oscillations. The long-term estimate uses Eq. (48). The estimate of Piriz et al. uses Eq. (49). The pole frequency is calculated by locating the pole in the complex plane.

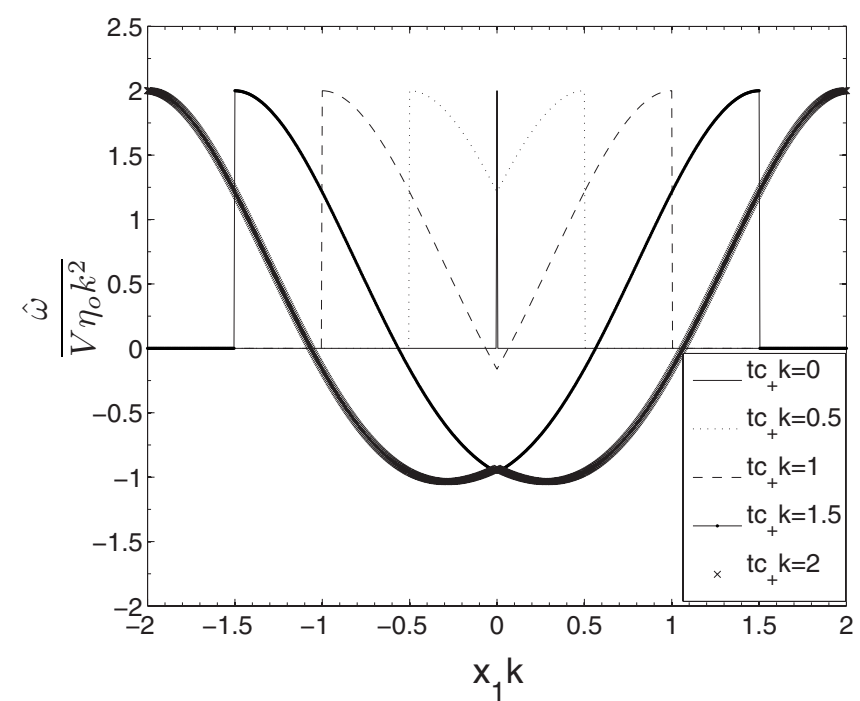

FIG. 9. Amplitude of vorticity at different times for $c_{-} / c_{+}=1$, $r=2$. Equal shear wave velocities produce a symmetric distribution of vorticity at each time.

fore, the growth rate of the interface is constant $[U(t, 0)$ $=A_{t} V \eta_{0} k$, with $r$ being the density ratio]. This growth rate is observed for our solution at $t=0^{+}$. After that moment, shear waves carry the vorticity away from the interface. Figures 9 and 10 show how the initial vorticity is carried off the interface for two cases: solids with equal shear wave velocity (Fig. 9) and solids with different shear wave velocities (Fig. 10).

To close the study of vorticity, we recall that another vortex sheet is produced by the jump in tangential velocity across the interface. Using the incompressibility condition (17), it is easy to derive the expressions for the tangential velocities from the normal velocity. This reveals that the behavior is similar to that of the growth rate (the parametric regions of oscillatory and decaying behavior are the same).

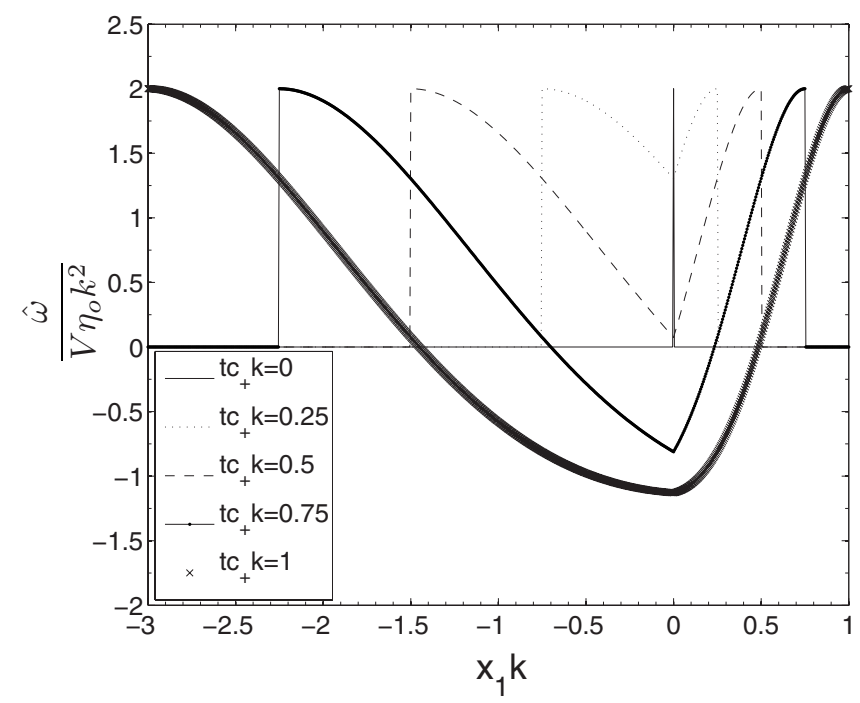

FIG. 10. Amplitude of vorticity at different times for $c_{-} / c_{+}=3$, $r=2$. Vorticity is carried off the interface faster in the material with higher wave velocity. 


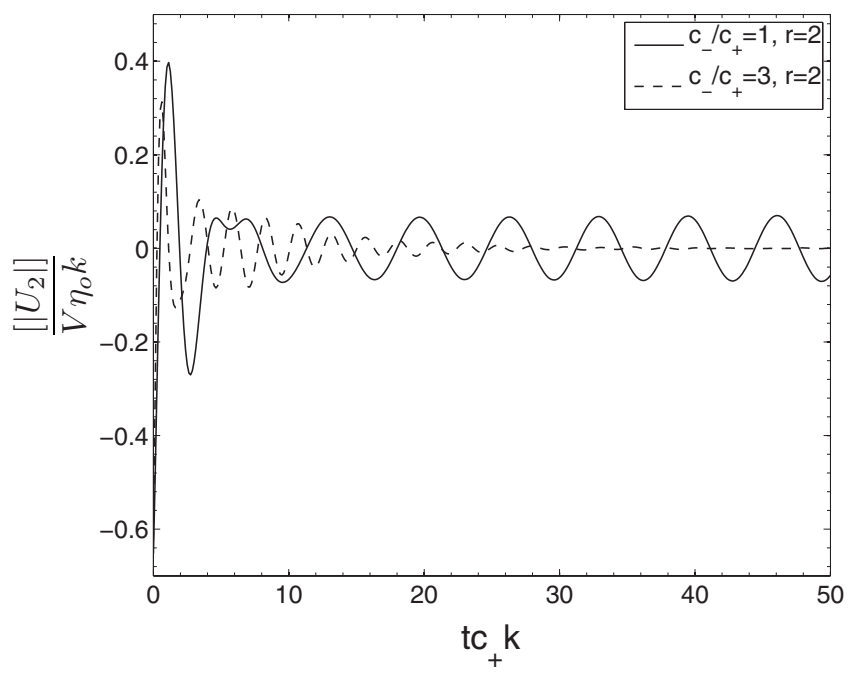

FIG. 11. Evolution of the tangential velocity jump across the interface for two different sets of parameters. The first set (continuous line) corresponds to an oscillatory long-term behavior while the second one (dashed line) corresponds to a decaying behavior.

Figure 11 shows the evolution of the jump in time for two different sets of parameters (case $1: c_{-} / c_{+}=1$ and $r=2$; case 2: $c_{-} / c_{+}=3$ and $\left.r=2\right)$.

\section{CONCLUSIONS}

An analytical approach to the Richtmyer-Meshkov flow for elastic solids allowed us to simply determine the parametric dependence of the problem. The incompressible and linear assumptions reduce the number of parameters in consideration to two: the shear wave velocity ratio and the density ratio. Then, the parameter space was explored with ease despite the difficulty of the expressions encountered.

Our analysis reveals the following conclusions concerning the amplitude and rate of growth of the interface between two elastic solids with frictionless contact:

(1) Solutions show a transitory period close to $t=0$ followed by a regular behavior. The rate of growth of the interface at $t=0$ corresponds to the Richtmyer-Meshkov result for fluids.

(2) Two different patterns are identified for the long-time behavior. The first one is purely oscillatory and appears when the two solids in contact have a very similar shear wave velocity or when one of the solids has no shear strength. The second behavior is described as an oscillatory decay that parametrically varies in oscillation frequency and rate of decay. As the ratio $c_{-} / c_{+}$separates from 1 , the decaying rate increases and then decreases as $c_{-} / c_{+}$becomes very large or very small, tending to the asymptotic oscillatory behavior when $c_{-} / c_{+} \rightarrow 0$ or $c_{-} / c_{+} \rightarrow \infty$.

(3) While the model of [8] accurately predicts the oscillation frequency of the interfacial waves, it incorrectly predicts the long-time amplitude. The amplitude should be computed using the full expression or performing a more accurate longtime approximation considering true pole locations and the branch cuts of the Laplace transform.
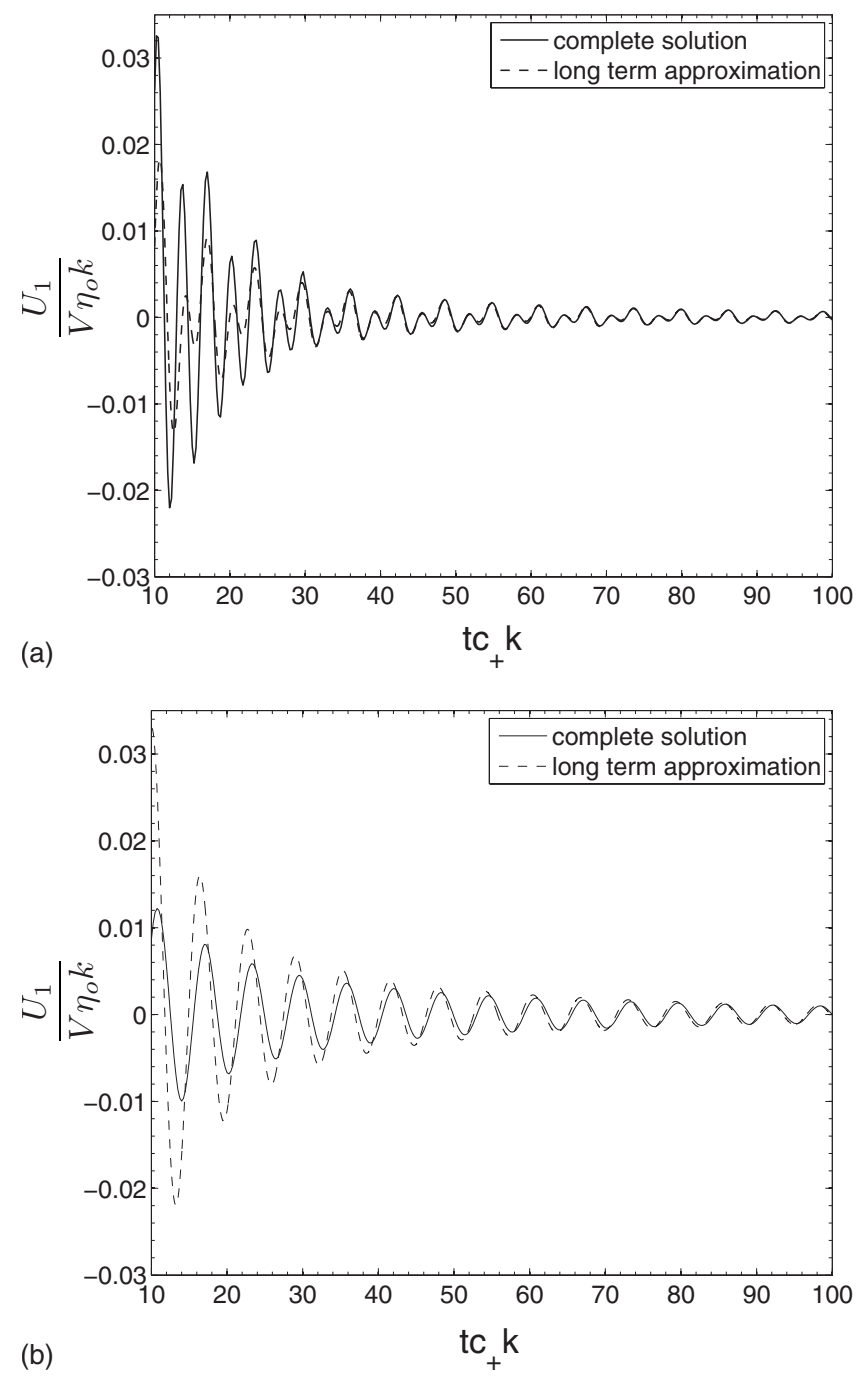

FIG. 12. Contribution of the integral over the branch cuts to the growth rate using a numerical quadrature method to integrate the growth rate function and using the first term of the approximation around the branch points.

We have shown the second-order wave equation with the shear wave velocity governing the vorticity within each material. Shear waves carry the initial vorticity deposited by the impulse at the interface at $t=0$ to the interior of the solid and away from interface. The tangential slip velocity at the interface either oscillates or decays while oscillating, as determined by the nature of the interfacial wave $\eta\left(t, x_{2}\right)$.

\section{ACKNOWLEDGMENTS}

We want to acknowledge M. Lombardini for his valuable comments on this paper. This material is based upon work supported by the Department of Energy National Nuclear Security Administration under Award No. DE-FC5208NA28613.

\section{APPENDIX: APPROXIMATION FOR THE GROWTH RATE USING LINEARIZATION AROUND BRANCH CUTS}

The symmetry of the problem allows us to only consider the integrals around the branch cuts located at $\operatorname{Im}(s)>0$, 
multiply the result by 2 , and take the real part of the expression,

$$
\frac{\partial \hat{\eta}}{\partial t}=\widehat{u_{1}}\left(t, x_{1}=0\right)=u_{\text {poles }}(t, 0)+\sum_{j=1}^{4} u_{b j}(t, 0),
$$

where $u_{\text {poles }}$ is the contribution of the residues of the poles to the normal velocity and leading-order branch-cut contributions $u_{b j}$ are

$$
\begin{aligned}
& u_{b 1}(t, 0)=-\frac{\sqrt{c_{-} k} \sqrt{2} e^{i c_{-} k t-i \pi / 4}}{\sqrt{\pi} t^{3 / 2}} K_{1}, \\
& u_{b 2}(t, 0)=-\frac{\sqrt{c_{+} k} \sqrt{2} e^{i c_{+} k t-i \pi / 4}}{\sqrt{\pi} t^{3 / 2}} K_{2} .
\end{aligned}
$$

For $c_{-}>c_{+}$,

$$
\begin{aligned}
& u_{b 3}(t, 0)=-\frac{i \sqrt{c_{-}\left(c_{-}^{2}-c_{+}^{2}\right) k^{3}} \sqrt{2} e^{i c_{-} k t-i \pi / 4}}{\sqrt{\pi} t^{3 / 2}} K_{3}, \\
& u_{b 4}(t, 0)=-\frac{\sqrt{c_{+}\left(c_{-}^{2}-c_{+}^{2}\right) k^{3}} \sqrt{2} e^{i c_{+} k t-i \pi / 4}}{\sqrt{\pi} t^{3 / 2}} K_{4} .
\end{aligned}
$$

For $c_{-}<c_{+}$,

$$
\begin{aligned}
& u_{b 3}(t, 0)=-\frac{\sqrt{c_{-}\left(c_{+}^{2}-c_{-}^{2}\right) k^{3}} \sqrt{2} e^{i c_{-} k t-i \pi / 4}}{\sqrt{\pi} t^{3 / 2}} K_{3}, \\
& u_{b 4}(t, 0)=-\frac{i \sqrt{c_{+}\left(c_{+}^{2}-c_{-}^{2}\right) k^{3}} \sqrt{2} e^{i c_{+} k t-i \pi / 4}}{\sqrt{\pi} t^{3 / 2}} K_{4},
\end{aligned}
$$

where coefficients $K_{i}$ are

$$
\begin{gathered}
K_{1}=\frac{4 c_{-}^{2}(1+r)\left[-48 c_{-}^{2} c_{+}^{6} r^{2}+32 c_{+}^{8} r^{2}-8 c_{-}^{6} c_{+}^{2} r(1+r)+c_{-}^{8}(1+r)^{2}+8 c_{-}^{4} c_{+}^{4} r(1+3 r)\right]}{k^{2}\left[-16 c_{+}^{6} r^{2}-8 c_{-}^{4} c_{+}^{2} r(1+r)+c_{-}^{6}(1+r)^{2}+8 c_{-}^{2} c_{+}^{4} r(1+3 r)\right]^{2}}, \\
K_{2}=\frac{4 c_{+}^{2} r(1+r)\left[32 c_{-}^{8}-48 c_{-}^{6} c_{+}^{2}-8 c_{-}^{2} c_{+}^{6}(1+r)+c_{+}^{8}(1+r)^{2}+8 c_{-}^{4} c_{+}^{4}(3+r)\right]}{k^{2}\left[-16 c_{-}^{6}-8 c_{-}^{2} c_{+}^{4}(1+r)+c_{+}^{6}(1+r)^{2}+8 c_{-}^{4} c_{+}^{2}(3+r)\right]^{2}}, \\
K_{3}=\frac{32 c_{-}^{2} c_{+}^{3} r(1+r)\left[-4 c_{-}^{2} c_{+}^{2} r+4 c_{+}^{4} r+c_{-}^{4}(1+r)\right]}{k^{3}\left[-16 c_{+}^{6} r^{2}-8 c_{-}^{4} c_{+}^{2} r(1+r)+c_{-}^{6}(1+r)^{2}+8 c_{-}^{2} c_{+}^{4} r(1+3 r)\right]^{2}}, \\
K_{4}=\frac{32 c_{-}^{3} c_{+}^{2} r(1+r)\left[4 c_{-}^{4}-4 c_{-}^{2} c_{+}^{2}+c_{+}^{4}(1+r)\right]}{k^{3}\left[-16 c_{-}^{6}-8 c_{-}^{2} c_{+}^{4}(1+r)+c_{+}^{6}(1+r)^{2}+8 c_{-}^{4} c_{+}^{2}(3+r)\right]^{2}} .
\end{gathered}
$$

The results are quite accurate even using only the first term (the term in $1 / t^{3 / 2}$ ) of the approximation. Normally this approximation matches the complete integration result earlier when the two shear wave velocities are very different from each other. Figures 12(a) and 12(b) show a comparison of both results (actual and approximate) for $c_{-} / c_{+}=2$ and $r=2$, and $c_{-} / c_{+}=1$ and $r=2$, respectively.
[1] R. D. Richtmyer, Commun. Pure Appl. Math. 13, 297 (1960).

[2] E. E. Meshkov, NASA Technical Transcriptions No. F-13, 074, 1970 (unpublished).

[3] M. Brouillette, Annu. Rev. Fluid Mech. 34, 445 (2002).

[4] J. D. Lindl, R. L. McCrory, and E. M. Campbell, Phys. Today 45(9), 32 (1992).

[5] D. Arnett, Astrophys. J. 127, 213 (2000).

[6] V. Wheatley, D. I. Pullin, and R. Samtaney, Phys. Rev. Lett. 95, 125002 (2005).

[7] J. N. Plohr and B. J. Plohr, J. Fluid Mech. 537, 55 (2005).
[8] A. R. Piriz, J. J. López Cela, N. A. Tahir, and D. H. H. Hoffmann, Phys. Rev. E 74, 037301 (2006).

[9] B. J. Plohr and D. H. Sharp, Z. Angew. Math. Phys. 49, 786 (1998).

[10] A. R. Piriz, J. J. López Cela, O. D. Cortázar, N. A. Tahir, and D. H. H. Hoffmann, Phys. Rev. E 72, 056313 (2005).

[11] G. Terrones, Phys. Rev. E 71, 036306 (2005).

[12] C. M. Bender and S. A. Orszag, Advanced Mathematical Methods for Scientists and Engineers (McGraw Hill, New York, 1978). 